\title{
Emerging role of carfilzomib in treatment of relapsed and refractory lymphoid neoplasms and multiple myeloma
}

This article was published in the following Dove Press journal:

Core Evidence

| April 201 I

Number of times this article has been viewed

\section{Salvia Jain \\ Catherine Diefenbach \\ Jasmine Zain \\ Owen A O'Connor}

NYU Cancer Institute, Division of Hematology and Medical Oncology, NYU Langone Medical Center, New York, NY, USA
Correspondence: Catherine Diefenbach Department of Medicine, Division of Hematology and Medical Oncology, NYU Cancer Institute, NYU Langone Medical Center, 7th floor, 160 East 34th Street New York, NY 10016, USA

$\mathrm{Tel}+|2| 273 \mid 5670$

Fax + I $21273 \mid 5540$

Email catherine.diefenbach@nyumc.org
Abstract: Proteasome inhibition forms the cornerstone of antimyeloma therapy. The first-in-class proteasome inhibitor, bortezomib, either alone or in combination with other chemotherapeutic agents, induces high overall response rates and response qualities in patients with clinically and molecularly defined high-risk disease. However, resistance to bortezomib and neurotoxicity associated with the treatment remain challenging issues. Carfilzomib is a novel, well tolerated, irreversible proteasome inhibitor with minimal neurotoxicity. Carfilzomib demonstrates promising activity in myeloma patients who are refractory to bortezomib and immunomodulatory agents. This review focuses on the pharmacology, safety, and efficacy of carfilzomib for the treatment of multiple myeloma in bortezomib-naïve and bortezomibexposed populations.

Keywords: carfilzomib, multiple myeloma, pharmacology, safety, efficacy

Core evidence clinical impact summary for carfilzomib in multiple myeloma (MM)

\begin{tabular}{|lll|}
\hline Outcome measure & Evidence & Implications \\
\hline $\begin{array}{l}\text { Disease-oriented evidence } \\
\text { Response rate }\end{array}$ & Substantial & $\begin{array}{l}\text { Patients with refractory MM } \\
\text { have partial and complete } \\
\text { response to drug }\end{array}$ \\
Improvement in quality of life & Substantial & $\begin{array}{l}\text { Patients had minimal to no } \\
\text { development of peripheral } \\
\text { neuropathy }\end{array}$ \\
Patient-oriented evidence & Moderate & $\begin{array}{l}\text { Grade } 3 \text { and } 4 \text { toxicities were } \\
\text { observed but infrequently led } \\
\text { to dose limitations }\end{array}$ \\
Dyspnea & Absolute 6\% increase in & $\begin{array}{l}\text { It may affect long-term } \\
\text { compliance if the agent is } \\
\text { to be used routinely }\end{array}$ \\
the incidence of dyspnea in & patients treated with ticagrelor \\
Economic evidence & Unknown & $\begin{array}{l}\text { The drug has not yet been } \\
\text { approved by US FDA for use } \\
\text { in relapsed/refractory MM }\end{array}$ \\
\hline
\end{tabular}

\section{Introduction}

Overall survival in multiple myeloma has improved significantly in the last decade. The emergence of new classes of drugs used as single agents or in combination has changed the natural history of the disease. Immunomodulatory drugs, such as thalidomide and lenalidomide, and proteasome inhibitors, such as bortezomib, when 
administered with conventional chemotherapeutic agents, ${ }^{1-7}$ have begun to rival the benefits seen with autologous stem cell transplantation. However, the majority of patients (even those who respond) eventually develop resistance over time by mechanisms that remain obscure. Early reports indicate that increased and/or altered proteasome subunit expression may play a role in acquired drug resistance to bortezomib, a reversible proteasome inhibitor approved for the treatment of multiple myeloma in both frontline and relapsed or refractory settings. ${ }^{8,9}$ This appears to be especially the case for the $\beta 5$-proteasome subunit, which exhibits chymotrypsin-like activity and is the major target for bortezomib. Its overexpression and mutation have induced bortezomib resistance in several in vitro models. ${ }^{9-12}$ In addition to the acquired resistance, painful peripheral neuropathy is a common dose-limiting toxicity of bortezomib, limiting its use in many patients. Most recently, a subcutaneous dosing schedule of bortezomib has been developed which appears to be associated with a substantially lower maximum concentration of exposure, with a roughly equivalent area under the curve of exposure and efficacy in patients with multiple myeloma. ${ }^{13}$ Interestingly, this alternative route of administration is also associated with a marked reduction in neuropathy, leading many to believe that the high maximum concentration exposure of bortezomib may be the primary basis for the neuropathy. It is also unclear whether this toxicity can be attributed to a host of alternative off-target effects, given the pleiotropic properties associated with proteasome inhibition. Collectively, these findings suggest the need for alternative proteasome inhibitors with an improved safety profile that may affect the proteasome in an irreversible fashion. Carfilzomib (formerly PR-171) is a novel proteasome inhibitor of the epoxyketone class that is structurally and mechanistically distinct from bortezomib. ${ }^{14}$ It is an irreversible inhibitor of the proteasome that seems to be more selective for chymotrypsin-like protease, with less affinity for the trypsin and caspase-like proteases in the $26 \mathrm{~S}$ proteasome. Preclinical and early-phase clinical studies of carfilzomib in patients with hematologic malignancies and multiple myeloma show promise.

\section{Chemical structure and biochemical pharmacology}

The ubiquitin-proteasome system pathway is the major nonlysosomal process responsible for maintaining cellular protein homeostasis via timely degradation of most intracellular proteins. Consequently, its inhibition affects numerous signaling pathways that regulate fundamental cellular functions, such as signal transduction, cell cycle progression (eg, cyclins), apoptosis (eg, Noxa, Bcl2/Bax), and the stress response (for example, misfolded and partially assembled proteins). Importantly, cancer cells appear to be particularly dependent on these proteasome-regulated homeostatic pathways. ${ }^{15-18}$ For a protein to be recognized by the proteasome, ubiquitin, a 76-residue polypeptide, must first be conjugated to the target protein. This process is carried out by a cascade of enzymes, including the ubiquitin-activating enzyme, the ubiquitin-conjugating enzyme, and ubiquitin ligase, which ultimately ubiqutinylates the target protein. Ubiquitin ligase binds to the target protein and interacts with the ubiquitin-conjugating enzyme to attach covalently a polyubiquitin chain of at least four units that flags the protein for destruction by the proteasome. This ubiquitin tagging earmarks the target protein for the $19 \mathrm{~S}$ regulatory cap, where, in an ATP-dependent fashion, the protein is converted to its primary structure. Once denatured, the protein is internalized into the core of the $26 \mathrm{~S}$ proteasome, where it undergoes proteolytic degradation via the proteases contained in the $\beta$-ring of the proteasome. The $26 \mathrm{~S}$ proteasome is comprised of a $20 \mathrm{~S}$ core containing three active enzymatic sites, including the chymotrypsin-like $(\beta 5)$, trypsin-like $(\beta 2)$, and post-glutamyl peptide hydrolase-like (caspase-like, $\beta 1$ ) activities, plus a $19 \mathrm{~S}$ regulatory cap at either end. ${ }^{19}$ Of these proteases, the chymotrypsin-like activity of the proteasome is most sensitive to inactivation. ${ }^{20,21}$

The immunoproteasome (20Si) is a specialized type of proteasome that is mainly found in monocytes and lymphocytes, and is known to be important for generating certain peptide antigens for major histocompatibility complex class I presentation. Upon stimulation with interferon-gamma or tumor necrosis factor-alpha, a change occurs in the protease subunit composition, leading to the replacement of $\beta 5$,

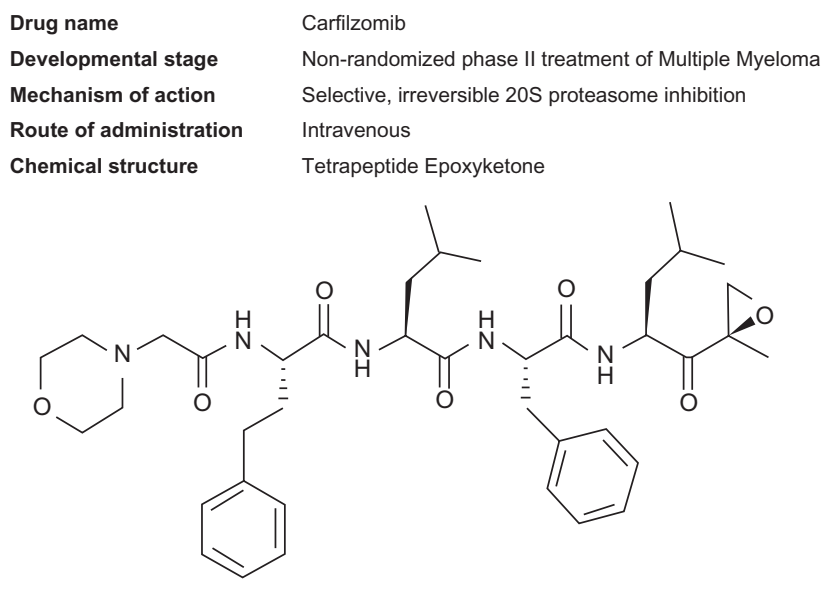

Figure I Carfilzomib: Structure and biochemical features. 


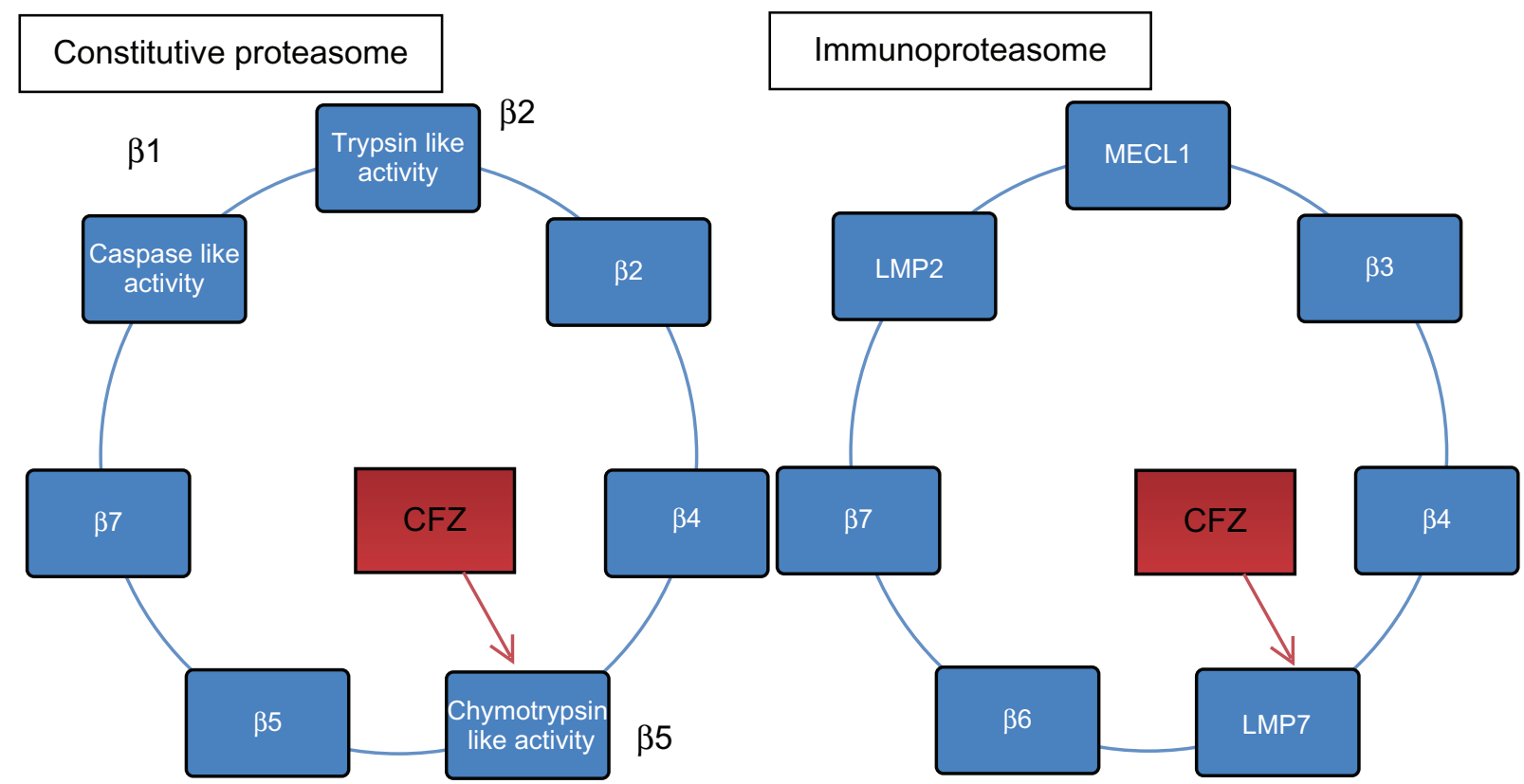

Figure 2 Cross sectional view of the carfilzomib binding site in the $20 \mathrm{~S}$ proteasome and immunoproteasome.

$\beta 2$, and $\beta 1$ by low molecular mass polypeptide 7 (LMP7), also known as $\beta 5 \mathrm{i}$, multicatalytic endopeptidase complex subunit (MECL1), also known as $\beta 2 \mathrm{i}$, and LMP2, also known as $\beta 1$ i. Preferential expression of $20 \mathrm{Si}$ has also been observed in multiple myeloma, suggesting that specific immunoproteasome inhibition could be a promising therapeutic strategy as well. ${ }^{22}$ Bortezomib and carfilzomib both appear to target the constitutive proteasome and immunoproteasome indiscriminately.

Carfilzomib is a structural analog (Figure 1) of the microbial natural product, epoxomicin 3 , that was initially identified for its antitumor activity and subsequently shown to be a potent inhibitor of the proteasome. ${ }^{14,21}$ Carfilzomib selectively inhibits the chymotrypsin-like activity of the $20 \mathrm{~S}$ proteasome and displays equivalent potency against $\beta 5$ and LMP7, and has minimal cross-reactivity with other protease classes (Figure 2). Proteasome inhibition by carfilzomib is mechanistically irreversible, ${ }^{14,22-24}$ and requires new protein synthesis for recovery of cellular proteasome activity. Consequently, the proteasome inhibition by carfilzomib is more sustained compared with that of the slowly reversible inhibitor, bortezomib. ${ }^{14}$ The epoxybutane pharmacophore of carfilzomib exhibits a high level of specificity for the $\mathrm{NH}_{2}$-terminal threonine residue that catalyzes enzymatic activity in each of the proteolytic active sites within the proteasome. ${ }^{23}$ Carfilzomib shows minimal activity against off-target enzymes, including serine proteases. ${ }^{25}$ In contrast, the boronate pharmacophore of bortezomib reacts with active-site serine residues found in

Table I Key biochemical and pharmacologic differences between major proteasome inhibitors in clinical use

\begin{tabular}{|c|c|c|c|}
\hline Properties & Bortezomib & Carfilzomib & NPI-0052 \\
\hline Active moiety & Dipeptide boronic acid analog & Peptide epoxyketone & $\begin{array}{l}\text { Nonpeptide bicyclic @-lactam } \\
\beta \text {-lactone }\end{array}$ \\
\hline $20 \mathrm{~S}$ proteasome & $\beta 5$ mainly & $\beta 5$ mainly & $\beta 5$ and $\beta 2$ \\
\hline$(20 \mathrm{Si})$ & LMP7 and $\beta I$ & LMP7 & Not reported \\
\hline \multicolumn{4}{|l|}{$\mathrm{IC}_{50} \mathrm{~S}(\mathrm{nM})$} \\
\hline Chymotrypsin & $2.4-7.9$ & 6 & 3.5 \\
\hline Trypsin & $590-4200$ & 3600 & 28 \\
\hline Caspase & $24-74$ & 2400 & 430 \\
\hline $\mathrm{IC}_{50}$ values across MM cell line RPMI-8226 (nM) & 5.7 & 5 & 9.1 \\
\hline Binding kinetics & Slowly reversible & Irreversible & Irreversible \\
\hline Half-life & I 10 minutes & $<30$ minutes & $<10-15$ minutes \\
\hline $\begin{array}{l}\text { Maximal percent of proteasome inhibition at } \\
\text { maximum tolerated dose }\end{array}$ & $65 \%-75 \%$ & $>80 \%$ & $100 \%$ \\
\hline
\end{tabular}

Abbreviations: LMP7, low molecular mass polypeptide 7; MM, multiple myeloma; $\beta$ I, $\beta$ I-proteasome subunit; $\beta 2$, $\beta 2$-proteasome subunit; $\beta 5$, $\beta 5$-proteasome subunit. 
serine proteases including chymotrypsin, cathepsins A and $\mathrm{G}$, elastase, and chymase, ${ }^{26,27}$ albeit at concentrations higher than required to inhibit the proteasome. Parlati et al confirmed the above observations, and recently demonstrated that 20Si is a major form of the proteasome expressed in hematopoietic cells, including multiple myeloma CD $138^{+}$tumor cells. ${ }^{28}$ Although specific inhibition of either LMP7 or $\beta 5$ alone was insufficient to produce an antitumor response, inhibition of all proteasome subunits was cytotoxic to both hematopoietic tumor cells and peripheral blood mononuclear cells. However, selective inhibition of both $\beta 5$ and LMP7 was sufficient for the antitumor effect in multiple myeloma, non-Hodgkin's lymphoma, and leukemia cells, while exhibiting minimal toxicity towards nontransformed cells. In multiple myeloma, chymotrypsin-like inhibition alone was sufficient to induce apoptosis. Differences between the major proteasome inhibitors with respect to the biochemical and pharmacological properties are listed in Table 1.

\section{Preclinical activity}

\section{In vitro activity in multiple myeloma}

Carfilzomib has been shown to induce apoptosis, cell cycle arrest, and activation of stress response pathways in a variety of human tumor cell lines, including multiple myeloma, Burkitt's lymphoma, acute lymphoblastic leukemia, B cell non-Hodgkin's lymphoma, colorectal adenocarcinoma, pancreatic cancer, and lung cancer. ${ }^{14}$ Demo et al have demonstrated that carfilzomib is more cytotoxic than bortezomib in cell culture following brief treatment, and that hematologic tumor cells exhibit the greatest sensitivity to brief exposure, whereas solid tumor cells and nontransformed cells were less sensitive to such treatments. There was no difference in the onset of proteasome inhibition between carfilzomib and bortezomib, eliminating the possibility that cell permeability could be a possible explanation for the difference in cytotoxicity. Extended exposure to carfilzomib (from 1-6 hours) typically produces greater cytotoxicity due to a time-dependent increase in proteasome inhibition and suppression of proteasome recovery.

Kuhn et al confirmed the potent and specific inhibition of the chymotrypsin subunit of the proteasome by carfilzomib, resulting in accumulation of the ubiquitinated substrates. They also demonstrated inhibition of the immunoproteasome by carfilzomib. ${ }^{29}$ Carfilzomib inhibited the proliferation of both interleukin-6-dependent ANBL-6 and KAS-6 multiple myeloma cell lines, as well as interleukin-6-independent lines, such as RPMI 8226 and U266, in a concentrationdependent and time-dependent manner. Greater antiprolifera- tive effects were seen across previously untreated multiple myeloma cell lines compared with bortezomib. Carfilzomib also overcame both primary and secondary resistance to bortezomib in both cell lines and patient-derived clinical samples. This observation was made in dexamethasoneresistant and melphalan-resistant cell lines, although the doxorubicin-resistant cell line did not respond to carfilzomib unless the cell line was pretreated with verapamil, a $\mathrm{P}$ glycoprotein inhibitor. In order to mimic the in vivo pharmacokinetic profile of proteasome inhibitor treatment, RPMI 8226 cells were treated with a one-hour pulse of drug followed by a washout. Accumulation of ubiquitin bound proteins and proapoptotic Bax, a proteasome target, were observed up to 48 hours after treatment, suggesting durable proteasome inhibition by carfilzomib. Inhibition of cell proliferation resulted in apoptosis which was associated with activation of c-Jun-N-terminal kinase, mitochondrial membrane depolarization, release of cytochrome c, and activation of both intrinsic and extrinsic caspase pathways. Both continuous and pulse administration of carfilzomib inhibited proliferation and induced programmed cell death in patientderived multiple myeloma cells and neoplastic cells.

In another recent study, Suzuki et al demonstrated that a bortezomib-resistant human colorectal adenocarcinoma cell line (HT-29) demonstrated high basal levels of proteasome catalytic activity (7-11-fold) compared with a bortezomibsensitive cell line.$^{30}$ Four hours after exposure to bortezomib, the resistant cells exhibited rapid recovery of proteasome activity relative to the sensitive cells, although carfilzomib induced prolonged inhibition of the proteasome in both bortezomib-sensitive and bortezomib-resistant cells, providing a possible explanation of its clinical activity in both bortezomib-naïve and bortezomib-treated patients. Resistant cells did not recover their proteasome activity to levels seen in parental cells at 24 hours after a brief exposure to bortezomib. This suggested alterations in proteasome assembly as a possible mechanism for the resistance to bortezomib. Sequence analysis of these resistant cells demonstrated a mutation in the propeptide region of the $\beta 5$ subunit of the proteasome (Arg24Cys). This region is required for proper proteasome assembly, and has previously been identified at a high frequency in myeloma patients. ${ }^{31}$ Suzuki et $\mathrm{al}^{30}$ also identified a novel mutation in LMP7 that might be responsible for rapid and efficient recovery of the proteasome and may explain the clinical resistance to bortezomib seen in myeloma patients. Carfilzomib was able to overcome bortezomib resistance in these cells due to prolonged irreversible inhibition of the proteasome. Collectively, these in vitro data highlight the 
potential of carfilzomib to overcome proliferation and induce apoptosis in both bortezomib-naïve and bortezomib-treated multiple myeloma cells without enhanced toxicity.

In order to evaluate if the myeloma cells in the bone marrow were equally vulnerable to proteasome inhibition by carfilzomib compared with peripheral blood mononuclear cells, Trudel et al measured the proteasome activity in both populations of cells. ${ }^{32}$ They employed an enzymatic assay using a fluorogenic substrate for the chymotrypsin-like activity and an enzyme-linked immunosorbent assay to quantitate inhibition of chymotrypsin-like and 20Si subunits (LMP7 and MECL1). Their results demonstrated that carfilzomib inhibited proteasome activity in myeloma cells in the bone marrow to the same extent as that seen in the mononuclear cells in whole blood. These data established that evaluating the peripheral blood mononuclear cells is a reasonable surrogate marker for proteasome inhibition in tumors, at least for multiple myeloma involving the bone marrow.

\section{In vitro activity in acute myelogenous leukemia}

Stapnes et al demonstrated that carfilzomib, like bortezomib, inhibited autocrine-dependent and cytokine-dependent proliferation of primary acute myelogenous leukemia blasts when tested at nanomolar concentrations $(0.1-100 \mathrm{nmol} / \mathrm{L}){ }^{33}$ Carfilzomib enhanced apoptosis in acute myelogenous leukemia cells and decreased proliferation and viability of tumor cells when tested in combination with idarubicin and cytarabine. Antiproliferative effects were also seen in human acute lymphoblastic leukemia cells. These data demonstrate the wide spectrum of antitumor activities exhibited by carfilzomib across various hematologic malignancies other than multiple myeloma.

\section{In vitro activity in non-Hodgkin's lymphoma in combination with histone deacetylase inhibitors}

Dasmahapatra et al demonstrated that the activity of carfilzomib in both germinal-center B cell-like diffuse large B cell lymphoma and activated B cell-like diffuse large B cell lymphoma was enhanced with coadministration of vorinostat, a histone deacetylase inhibitor. ${ }^{34}$ Vorinostat increased the carfilzomib activity in diffuse large B cell lymphoma cells resistant or sensitive to bortezomib. This was mediated via increased mitochondrial injury, caspase activation, apoptosis through Jun NH2-terminal kinase and p38 mitogen-associated protein kinase activation. Abrogation of histone deacetylase inhibitor-mediated nuclear factor-kappa B activation,
AKT inactivation, and $\mathrm{Ku} 70$ acetylation, was also observed, which contributed to the synergistic activity. To date, this synergy has not been explored in preclinical models of multiple myeloma but supports investigation. Recently Rao et al reported that when mantle cell lymphoma cells were cotreated with a specific histone deacytelase-6 inhibitor, WT 161 , it disrupted hsp-90 function, abrogated aggresome formation, and sensitized the cells to lethal endoplasmic reticulum stress induced by carfilzomib. ${ }^{35}$ As already established with bortezomib, these observations highlight the potential of combining carfilzomib with other chemotherapeutics, especially histone deacetylase inhibitors.

\section{In vivo activity}

Demo et al demonstrated that carfilzomib was able to induce apoptosis in human tumor xenograft models of colorectal cancer, B cell lymphoma, and Burkitt's lymphoma, with daily dosing schedules that also induced high levels of proteasome inhibition. ${ }^{14}$ Importantly, Demo et al established that dosing for two consecutive days exhibited superior antitumor activity in xenograft tumors when compared with carfilzomib or bortezomib given in a split dosing schedule (day 1 and day 4) or on a higher-dose weekly schedule, suggesting that the improved efficacy with a consecutive day schedule could be due to inhibition of proteasome recovery between doses. Despite this intensive daily dosing that resulted in greater than $80 \%$ proteasome inhibition in most tissues, it was well tolerated without any excessive toxicity. These data led to the selection of the two consecutive day schedules for Phase I clinical trials.

\section{Pharmacokinetic and pharmacodynamic profile}

Studies in rodents and nonhuman primates have demonstrated that carfilzomib is rapidly cleared from the plasma compartment following intravenous bolus administration, with a terminal half-life in rats and monkeys of 15 and 7.2 minutes, respectively. ${ }^{36}$ Despite the rapid clearance, carfilzomib produced a prolonged dose-dependent inhibition of $20 \mathrm{~S}$ proteasome in all tissues except the brain. In rats and monkeys, carfilzomib was administered for either two or five consecutive days, followed by nine days of rest for two 14-day cycles. Doses that resulted in greater than $80 \%$ inhibition of proteasome activity in blood and other tissues were found to be well tolerated. ${ }^{14}$ It was also superior to the split dosing schedule (day 1 and day 4). Recovery of proteasome activity following repeated daily administrations was unchanged from that seen after a single dose. A transient thrombocytopenia 
was noted in both rats and monkeys following administration of carfilzomib, but neutrophil and lymphocyte counts remained unchanged. These observations established the rationale for the schedule-dependency and likely antitumor activity, and formed the basis for the design of the first Phase I dose escalation study. ${ }^{37}$ In this first Phase I experience by O'Connor et al, 29 patients with refractory or relapsed multiple myeloma, non-Hodgkin's lymphoma, Waldenstrom's macroglobulinemia, or Hodgkin's lymphoma were enrolled. Patients received carfilzomib every day for five consecutive days every 14 days at doses of 1.2, 2.4, 4.6, 8.4, 11, 15, and $20 \mathrm{mg} / \mathrm{m}^{2}$. No dose-limiting toxicities were observed in the $1.2-15 \mathrm{mg} / \mathrm{m}^{2}$ cohorts. However, at the $20 \mathrm{mg} / \mathrm{m}^{2}$ dose, grade 3 febrile neutropenia, and grade 4 thrombocytopenia (two patients of five in that cohort) were noted, establishing $15 \mathrm{mg} / \mathrm{m}^{2}$ as the maximum tolerated dose. Antitumor activity was observed at doses greater than $11 \mathrm{mg} / \mathrm{m}^{2}$, including one unconfirmed complete response (mantle cell lymphoma), one partial response (multiple myeloma), and two minimal responses (multiple myeloma and Waldenstrom's macroglobulinemia). The mean maximum concentration for the $15 \mathrm{mg} / \mathrm{m}^{2}$ dose was $325.9(83.7-620) \mathrm{ng} / \mathrm{mL}$, time to maximum concentration was $5.8(5-7)$ minutes, area under the curve was $9728(1616-28,426)$ ng-mL/hour, elimination half-life was 28.9 (6.8-95.5) minutes, systemic clearance was $7054(950-18,511) \mathrm{mL} / \mathrm{min}$ and volume of distribution at steady state was 942 (1417-4603) L. Clearance was rapid, with an elimination half-life of less than 30 minutes, in accordance with preclinical data and through renal and biliary excretion. Although the peak concentration and area under the curve increased with dose, the increase was not dose-proportional. Mild to moderate fatigue $(48 \%, \mathrm{n}=14)$, nausea $(48 \%, \mathrm{n}=14)$, diarrhea $(35 \%, \mathrm{n}=10)$, dyspnea $(28 \%, \mathrm{n}=8)$, pyrexia $(28 \%, \mathrm{n}=8)$, hypoesthesia $(28 \%$, $\mathrm{n}=8)$, headache $(24 \%, \mathrm{n}=7)$, constipation $(21 \%, \mathrm{n}=6)$, peripheral edema $(24 \%, n=7)$, and anemia were reported. Forty-eight percent $(n=14)$ of the patients experienced grade 3-4 toxicity, but no patient experienced grade 3-4 peripheral neuropathy, despite inclusion of patients with symptomatic or pre-existing neuropathy. These data were among the first to establish the reduced incidence of neuropathy associated with carfilzomib.

Pharmacodynamic studies in this trial showed that carfilzomib induced a dose-dependent inhibition of $20 \mathrm{~S}$ chymotrypsin-like proteasome activity in whole blood and peripheral blood mononuclear cells within one hour of drug administration on day 1 . More than $75 \%$ inhibition of chymotrypsin-like activity was achieved in whole blood and peripheral blood mononuclear cells with the first dose of carfilzomib $\geq 11 \mathrm{mg} / \mathrm{m}^{2}$. With repeat dosing, cumulative proteasome inhibition was observed in whole blood and peripheral blood mononuclear cells within one hour after the fifth consecutive day of dosing in the highest dose groups, which was also well tolerated. Activity generally returned to the baseline in peripheral blood mononuclear cells during the nine-day rest period, but only partially recovered in whole blood due to the inability of erythrocytes to synthesize new proteasome protein. No unexpected adverse events were associated with the extended five-day dosing with carfilzomib. This study demonstrated activity of carfilzomib in multiple hematologic malignancies, including bortezomibresistant multiple myeloma, confirmed sustained inhibition of the chymotrypsin-like activity of the proteasome subunit, and established the drug and the schedule as safe.

In order to evaluate the efficacy, safety, and pharmacokinetic and pharmacodynamic profile of an intravenous bolus versus a protracted infusion (over 30 minutes) schedule of carfilzomib, a Phase Ib/II study (PX-171-007) was conducted by Rosen et al in patients with advanced metastatic solid tumors. $^{38,39}$ The Phase I bolus trial enrolled patients with solid tumors failing at least two prior treatments. Carfilzomib was given on days $1,2,8,9,15$, and 16 every 28 days for up to 12 cycles. During cycle 1 , day 1 and day 2 dosing in all cohorts was $20 \mathrm{mg} / \mathrm{m}^{2}$, with subsequent escalation to 20,27 , or $36 \mathrm{mg} / \mathrm{m}^{2}$ on day 8 . One patient who received the $36 \mathrm{mg} / \mathrm{m}^{2}$ dose on day 8 developed a dose-limiting toxicity (grade 3 fatigue). Hence $20 \mathrm{mg} / \mathrm{m}^{2}$ was selected as the Phase II dose. Fourteen patients were enrolled in the Phase I bolus trial, 20 patients in the Phase I infusion study and 65 patients in the Phase II study, which included 12 small cell lung cancers, 17 non-small cell lung cancers, 16 ovarian, 10 renal, and 24 other cancers. A separate treatment arm was added for patients with multiple myeloma in this category. Partial responses were seen in patients with renal cancer, non-small cell lung cancer, and multiple myeloma, whereas stable disease lasting for greater than 16 weeks was observed in patients with mesothelioma, ovarian, renal, cervical, endometrial, small cell lung, and non-small cell lung cancer. As noted in the earlier Phase I studies, no patients experienced greater than grade 1 peripheral neuropathy, even at the highest doses of carfilzomib administered (ie, $36 \mathrm{mg} / \mathrm{m}^{2}$ on day 8). These data established that carfilzomib was tolerated and equally efficacious as a bolus and infusion administration.

A second parallel Phase1 trial (PX-171-002) conducted by Alsina et al evaluated a consecutive day schedule for three consecutive weeks on a 28-day cycle (days 1 and 2, days 
8 and 9 , days 15 and 16 , every 28 days) at doses ranging from $1.2 \mathrm{mg} / \mathrm{m}^{2}$ to $27 \mathrm{mg} / \mathrm{m}^{2}$ in 37 patients with relapsed or refractory lymphoid neoplasms and multiple myeloma. ${ }^{40}$ They found that $15 \mathrm{mg} / \mathrm{m}^{2}$ was the minimum effective dose, with greater than $80 \%$ proteasome inhibition being achieved at this dose, with good tolerability. Five objective responses were observed in 16 patients (13 multiple myeloma, three mantle cell lymphoma) enrolled at the minimum effective dose or higher. Four patients with multiple myeloma attained a partial response, and one patient with multiple myeloma attained a minimal response. The responses were durable (134-392 days), and occurred in patients who relapsed following prior bortezomib, immunomodulatory agents like thalidomide and lenalidomide, and stem cell transplantation. A reversible grade 2 first-dose effect of increased creatinine at doses of $20 \mathrm{mg} / \mathrm{m}^{2}$ and higher was observed. Given the safety and efficacy of this schedule, it was chosen as the Phase II dose for patients with multiple myeloma and other solid tumors in refractory/relapsed settings.

\section{Clinical activity}

Given the encouraging safety profile and clinical activity observed in the Phase I studies, Phase II trials of carfilzomib were initiated in patients with relapsed and refractory multiple myeloma. In an open-label, multicenter trial, Jagannath et al demonstrated dramatic single agent activity of carfilzomib in patients with relapsed and refractory multiple myeloma. ${ }^{41}$ Forty-six patients with multiple myeloma, who had relapsed disease following at least two prior therapies, received carfilzomib at a dose of $20 \mathrm{mg} / \mathrm{m}^{2}$ intravenously on days 1, 2, 8, 9, 15 , and 16 every 28 days for up to 12 cycles. The median number of prior therapies was five (range 2-15), which included an anthracycline and alkylating agent, and 83\% had had a prior stem cell transplant. Patients received a median of three cycles of carfilzomib (range 1-12). Clinical response, defined as minimal response or greater, was seen in 10 patients $(26 \%)$, including five patients achieving a partial response and five patients attaining a minimal response. No patients achieved a complete remission. Five patients with bortezomib-refractory disease achieved a minimal response or a partial response. The median time to progression was 6.2 months, while the median duration of response was 7.4 months. This time to progression is similar to that observed with bortezomib in both the SUMMIT trial conducted in relapsed and refractory multiple myeloma patients (median time to progression seven months) and the APEX trial conducted in patients who had relapsed myeloma (median time to progression 6.2 months). Ten percent of the patients (four of 39) completed 12 cycles, highlighting that prolonged treatment with carfilzomib is possible. Eight of the 10 patients achieved a response during cycle 1, and peripheral neuropathy was noted in less than $10 \%$ of the patients, with one grade 3 neuropathy occurring in a patient with pre-existing grade 2 neuropathy. Common adverse events included fatigue (65\%), anemia (65\%), thrombocytopenia (46\%), neutropenia (20\%), nausea (37\%), upper respiratory infection (37\%), and diarrhea (33\%). Increased creatinine, both drug and nondrug-related, was seen in 15 of 46 patients (33\%), but treatment was discontinued in only three patients due to nephrotoxicity. Acute renal failure was documented in four patients $(9 \%)$, two $(4 \%)$ of whom also had possible tumor lysis syndrome, thus making it difficult to discern completely the effect of carfilzomib on renal function. This was the first Phase II clinical study of carfilzomib, which showed single-agent activity in a heavily pretreated, relatively bortezomib-resistant population of patients with relapsed or refractory multiple myeloma.

These encouraging results led to PX-171-003-A1, an open-label, Phase IIb trial conducted by the Multiple Myeloma Research Consortium. ${ }^{42}$ Two hundred and sixty-six patients with refractory multiple myeloma were included. Patients were required to have had at least two prior therapies, including bortezomib and either thalidomide or lenalidomide, and an alkylating agent. The enrolled patients had received a median of five prior lines of therapy (range 1-20 and a median of 13 antimyeloma agents). Of note, the majority of the patients had failed at least two prior bortezomibcontaining regimens. Patients received carfilzomib at $20 \mathrm{mg} / \mathrm{m}^{2}$ on days $1,2,8,9,15$, and 16 every 28 days in cycle 1 , and were dose-escalated to $27 \mathrm{mg} / \mathrm{m}^{2}$ on the same schedule thereafter for up to 12 cycles. The primary endpoint was overall response rate. An overall response rate of $24 \%$ with a median duration of response of 7.4 months (range 6.2-10.3) was observed. One complete response $(0.4 \%), 12$ very good partial responses $(4.7 \%), 48$ partial responses $(19 \%)$, and 32 minimal responses (12\%) were achieved. An additional eighty three $(32 \%)$ patients achieved stable disease for at least six weeks. Eleven percent of the patients completed the entire 12 cycles and went on into the extension study. The most common treatment-emergent adverse events $\geq$ grade 3 regardless of the relationship to study drug were hematologic, including thrombocytopenia (22\%), anemia (20\%), lymphopenia $(10 \%)$, pneumonia $(8 \%)$, neutropenia $(8 \%)$, fatigue $(7 \%)$, hyponatremia $(5 \%)$, and hypercalcemia (5\%). Although 206 (77\%) patients experienced grade 1 or 2 peripheral neuropathy at baseline, new onset peripheral neuropathy was infrequent. Greater than grade 3 neuropathy 
occurred in less than 1\% of patients. Analysis of this subset of patients with active peripheral neuropathy (grade 1 and 2) in this trial demonstrated that peripheral neuropathy had no impact on the depth or durability of responses, or on the tolerability of carfilzomib. This Phase II study demonstrated that single-agent carfilzomib can induce durable responses in heavily pretreated multiple myeloma patients who had failed multiple chemotherapy regimens, including those containing bortezomib and immunomodulatory agents. Significantly, even patients with baseline neuropathy tolerated prolonged treatment with carfilzomib, with minimal risk of exacerbation.

A second parallel Phase II multicenter study (PX-171-004) of carfilzomib was conducted by the Multiple Myeloma Research Consortium to determine its efficacy in bortezomibnaive relapsed multiple myeloma patients. ${ }^{43-46}$ Fifty-four patients received carfilzomib at a dose of $20 \mathrm{mg} / \mathrm{m}^{2}$ intravenously on days $1,2,8,9,15$, and 16 every 28 days for up to 12 cycles, while 19 patients received the escalated dose of $27 \mathrm{mg} / \mathrm{m}^{2}$ on the same schedule. The overall response rate was $46 \%$ ( 25 of 54 patients), which included one overall response rate, five very good partial responses, and 19 partial responses at the $20 \mathrm{mg} / \mathrm{m}^{2}$ dose. In addition, nine patients experienced a minimal response and 10 patients had stable disease. At the $27 \mathrm{mg} / \mathrm{m}^{2}$ dose, the overall response rate was 53\%, with one very good partial response and nine partial responses. The median duration of response was 8.8 months and the median time to progression was 7.6 months. The adverse events were similar to the previous studies, and included fatigue (59\%), nausea (41\%), dyspnea (36\%), anemia
(29\%), increased creatinine (31\%), and upper respiratory tract infection (31\%), with most $\leq$ grade 2 . Grade 3 and 4 adverse events which occurred in $5 \%$ or more patients were thrombocytopenia (9\%), fatigue (9\%), neutropenia $(7 \%)$, lymphopenia (7\%), anemia (5\%), pneumonia $(5 \%)$, and hyperglycemia (5\%). Dose reductions were rarely invoked, and the drug was well tolerated in patients with baseline renal insufficiency. Increased creatinine, irrespective of attribution, was seen in five patients $(16 \%)$, but treatment was discontinued in only one patient due to a renal adverse event. Subsequently, patients in this study were stratified into a highrisk category based on their Eastern Cooperative Oncology Group performance score, cytogenetic profile, and serum $\beta_{2}$ microglobulin, and responses of this subset were analyzed. Single-agent carfilzomib produced an overall response rate of $41 \%-54 \%$ in this high-risk group, highlighting its potential as a steroid-sparing agent in patients with multiple comorbidities. Comparison of the early-phase results of some of the proteasome inhibitors currently in clinical use in multiple myeloma are summarized in Table 2.

Jakubowiak et al recently evaluated the influence of disease cytogenetics in 79 patients enrolled in the PX-171-003 and PX-171-004 trials, in whom metaphase cytogenetics and/or fluorescence in situ hybridization analysis for del $13 \mathrm{q}, \mathrm{t}(4 ; 14)$ and $t(14 ; 16)$ chromosomal abnormalities were available. ${ }^{47}$ The response rate ( $\geq$ minimal response) for the entire group of patients was $40.5 \%$. Twenty-three of the 79 patients had at least one of the cytogenetic abnormalities. However, the presence of these cytogenetic lesions did not impact the response rate. Approximately $43 \%$ of patients with one or more genetic

Table 2 Comparison of the early-phase trials of major proteasome inhibitors in clinical use

\begin{tabular}{|c|c|c|c|}
\hline Outcome & Bortezomib & Carfilzomib & NPI-0052 \\
\hline Maximum tolerated dose/schedule & $\begin{array}{l}1.3 \mathrm{mg} / \mathrm{m}^{2} \text { on days I, } 4,8 \text {, and } \\
\text { II every } 2 \text { I days }\end{array}$ & $\begin{array}{l}20 \mathrm{mg} / \mathrm{m}^{2} \text { on days } \mathrm{I}, 2,8,9 \\
15 \text {, and } 16 \text { every } 28 \text { days }\end{array}$ & $\begin{array}{l}0.7 \mathrm{mg} / \mathrm{m}^{2} \text { on days } \mathrm{I}, 8 \text {, } \\
\text { and } \mathrm{I} 5 \text { every } 28 \text { days }\end{array}$ \\
\hline Dose-limiting toxicity & $\begin{array}{l}\text { Thrombocytopenia, } \\
\text { hyponatremia, fatigue }\end{array}$ & Fatigue & $\begin{array}{l}\text { Fatigue, mental status } \\
\text { change, loss of balance }\end{array}$ \\
\hline Phase & II, Richardson et al ${ }^{4}$ & II, Jagannath et $\mathrm{al}^{4 I}$ & I, Richardson et al ${ }^{78}$ \\
\hline ORR n (\%) & $67(35)$ & $61(24)$ & I (3.5) \\
\hline $\mathrm{n}$ & 193 & 266 & 27 \\
\hline $\mathrm{CR}$ or $\mathrm{nCR}$ & $19(10)$ & $\mathrm{I}(0.4)$ & None \\
\hline \multicolumn{4}{|l|}{ Patients (n, \%) } \\
\hline PR & $34(18)$ & $48(19)$ & I (3.5) \\
\hline \multicolumn{4}{|l|}{ Patients (n, \%) } \\
\hline Median duration of response & 12 months & 7.4 months & Not reported \\
\hline Median TTR & 1.3 months & I month & Not reported \\
\hline Median TTP & 7 months & Not reported & Not reported \\
\hline Grade 3 peripheral neuropathy (\%) & $12 \%$ & $<1 \%$ & None \\
\hline Developmental stage & Approved for MM and relapsed MM & Phase II & Phase I \\
\hline
\end{tabular}

Abbreviations: ORR, overall response rate, ie, $\geq \mathrm{PR}$; $\mathrm{CR}$, complete remission; $\mathrm{nCR}$, near complete remission; $\mathrm{n}$, number of patients enrolled in the trial; $\mathrm{PR}$, partial response; DoR, duration of response; TTP, time to progression; TTR, time to a first response; MM, multiple myeloma. 
lesions responded, compared with $39.3 \%$ of patients with no cytogenetic abnormality. The median time to progression was 203 days for all patients in this analysis, with a time to progression of 195 days for patients with one or more abnormalities, and 208 days for patients with none. Similar to what has been seen with bortezomib, these data appear to suggest that carfilzomib has efficacy even in patients with well established adverse prognostic factors in myeloma.

Given the preclinical evidence of synergy between carfilzomib and dexamethasone demonstrated by Kuhn et al, ${ }^{29}$ a Phase Ib study of carfilzomib (PX-171-006) in combination with lenalidomide and low-dose dexamethasone was conducted by Niesvizky et al in patients with relapsed and refractory multiple myeloma. ${ }^{48,49}$ The aim was to assess the safety and feasibility of this combination. Sixty-seven patients with a median of two prior therapies (including lenalidomide, thalidomide, bortezomib, stem cell transplant, alkylators, and anthracyclines) were enrolled. Carfilzomib was administered in doses ranging from $15 \mathrm{mg} / \mathrm{m}^{2}$ to $27 \mathrm{mg} / \mathrm{m}^{2}$ on days $1,2,8,9,15$, and 16 intravenously), lenalidomide in doses ranging from $10 \mathrm{mg}$ to $20 \mathrm{mg}$ orally on days 1 and 2 , and low-dose dexamethasone at a dose of $40 \mathrm{mg}$ orally on days $1,8,15$, and 22 in 28-day cycles. The regimen induced rapid responses within the first cycle in most patients. The overall response rate and clinical benefit response (defined as minimal response or better) for 29 evaluable patients was $59 \%$ and $72 \%$, respectively, which included a $21 \%$ overall response rate/near overall response rate $(n=6)$, a $17 \%$ very good partial response rate $(n=5)$, and a $21 \%$ partial response rate $(n=6)$. Forty-three of the 67 patients had remained on the study at the time when the results were reported. Grade 3 hematologic toxicity was minimal and reversible, ie, thrombocytopenia $(n=6)$, anemia $(n=4)$, and neutropenia $(n=6)$. Other toxicities, including fatigue and neuropathy, did not exceed grade 2 . Interestingly, the tolerability of the regimen allowed continued administration of the combination for up to 18 cycles, and prior exposure to bortezomib and immunomodulatory drugs did not preclude the patients from achieving a response.

Alsayed et al conducted a small trial exploring the coadministration of carfilzomib with other agents, such as dexamethasone, liposomal doxorubicin, thalidomide, cisplatin, and vorinostat, in a graded manner in 17 patients with advanced, refractory multiple myeloma and high-risk gene expression profiles. ${ }^{50}$ Seventeen heavily pretreated patients received carfilzomib $20 \mathrm{mg} / \mathrm{m}^{2}$ on days 1 and 2 , and $27 \mathrm{mg} / \mathrm{m}^{2}$ on days $8,9,15$, and 16 , along with dexamethasone $4 \mathrm{mg}$ with each dose of carfilzomib. Thirteen patients started the second cycle of carfilzomib at $36 \mathrm{mg} / \mathrm{m}^{2}$, along with dexamethasone ranging in dose from $4 \mathrm{mg}$ to $20 \mathrm{mg}$. Nine patients started the third cycle of carfilzomib at $36 \mathrm{mg} / \mathrm{m}^{2}$, along with dexamethasone, with the addition of thalidomide $100 \mathrm{mg}$ /day and doxorubicin $3 \mathrm{mg} / \mathrm{m}^{2} /$ day $\times 16$ days for one cycle. Four patients received a fourth cycle as in cycle 3 with added cisplatin at $2 \mathrm{mg} / \mathrm{m}^{2} /$ day for 16 days for one cycle. Two patients finished a fifth cycle with further addition of vorinostat $200 \mathrm{mg}$ /day. Responses included one overall response (cycle 1), three partial responses (two in cycle 2 , one in cycle 4), and four improvements. Toxicities attributed to carfilzomib were minor, with no evidence of bone marrow or kidney dysfunction or peripheral neuropathy. This study provides a platform for the use of carfilzomib in combination with other approved drugs without any enhanced toxicity.

Given the incidence of renal impairment in patients with myeloma, an organ dysfunction study (PX-171-005) was performed by Bardos et al.51,52 Thirty-nine patients were enrolled into the study. Ten patients with normal renal function, nine with mild renal insufficiency (creatinine clearance 50-79 $\mathrm{mL} / \mathrm{min}$ ), nine with moderate (creatinine clearance 30-49 $\mathrm{mL} / \mathrm{min}$ ), nine with severe renal insufficiency (creatinine clearance $<30 \mathrm{~mL} / \mathrm{min}$ ), and two patients on hemodialysis were included. These patients received carfilzomib at a dose of $15 \mathrm{mg} / \mathrm{m}^{2}$ in cycle 1 , escalating to a $20 \mathrm{mg} / \mathrm{m}^{2}$ schedule in cycle 2 and $27 \mathrm{mg} / \mathrm{m}^{2}$ in cycle 3 . Renal impairment did not affect the pharmacokinetic or pharmacodynamic profile across all categories of renal dysfunction. Carfilzomib was undetectable in plasma within three hours, and did not accumulate after cycle 2 . Of note, eight partial responses, five minimal responses, and 13 patients with stable disease were observed despite renal impairment. This study clearly establishes the safety and similar efficacy of carfilzomib administration in patients with marked renal insufficiency, a well known feature in multiple myeloma, without dose adjustments.

Collectively, these trials establish the significant activity and favorable safety profile of carfilzomib in patients with heavily treated myeloma. Carfilzomib appears to distinguish itself from bortezomib mostly with regard to its favorable toxicity profile with substantially less neurotoxicity. The results of major clinical trials performed to date with carfilzomib are summarized in Table 3.

\section{Side effect profile}

One of the major complications and the dose-limiting toxicity of agents active in multiple myeloma like bortezomib and thalidomide, is debilitating peripheral neuropathy. While 


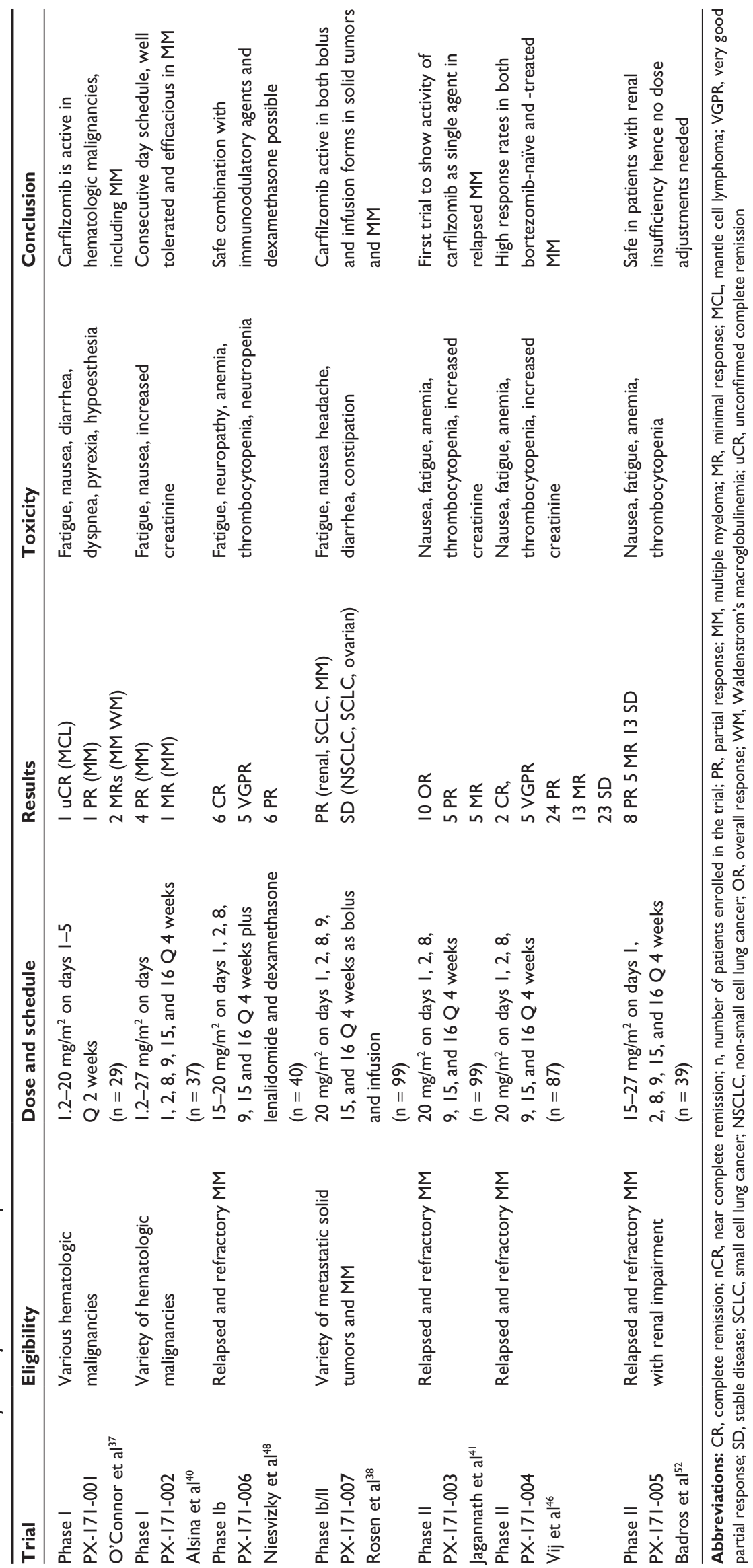


the exact mechanism behind this toxicity remains poorly understood, it appears that this may not be a class effect of proteasome inhibitors.

Vij et al evaluated the neuropathic effects of carfilzomib in patients enrolled in the Phase II trials (PX-171-003 and PX-171-004). ${ }^{53}$ Detailed neurological examinations and subjective reporting of peripheral neuropathy using the FACTGOG/Ntx subscale version 4 questionnaire were recorded every two cycles until discontinuation of the drug. At the time the results were reported, data were available for 136 patients. Seventy-three patients had peripheral neuropathy at baseline (64 with grade 1 and nine with grade 2) primarily attributed to prior use of thalidomide or bortezomib in the majority of cases. The median number of carfilzomib cycles received by the patients was 4.5 . Peripheral neuropathy was reported in $21(15 \%)$ patients, with 12 cases $(9 \%)$ attributed to carfilzomib. The incidence of grade 3 or greater peripheral neuropathy was only $2 \%(\mathrm{n}=3)$, while the incidence of grade 1 or grade 2 paresthesias and dysesthesias was $7 \%$ (in 10 patients only). None of the 136 patients required discontinuation or dose adjustments because of neurotoxicity, and there was no statistically significant change in FACT-GOG/Ntx scores before and after starting carfilzomib. These data clearly establish the favorable side effect profile of carfilzomib, which does not appear to require major dose or schedule modifications even in patients with pre-existing neuropathy. The precise reason why carfilzomib exhibits less neurotoxicity compared with bortezomib is unclear, with many attributing the differences to its selectivity and lack of off-target effects. An in vitro neurotoxicity model established to compare treatment-induced peripheral neuropathy between carfilzomib and bortezomib demonstrated that, after treatment with bortezomib, differentiated neuroblastoma cells showed a $40 \%$ reduction in neuron length compared with carfilzomib. ${ }^{52} \mathrm{HtrA} 2 / \mathrm{Omi}$, a mitochondrial serine protease with a role in neuron survival, has been confirmed as a target of bortezomib but not carfilzomib. It is interesting that the amount of proteasomal inhibition appears unrelated to the neuropathic effects, confirming that this is not a class effect of the proteasome inhibitors.

Aside from neurotoxicity, constitutional symptoms, such as fatigue, are seen in the majority of patients $(57 \%-66 \%)$ treated with carfilzomib, although the majority of these events are primarily less than grade 2, with grade 3 or 4 fatigue occurring in about $9 \%$ of patients. None of these toxicities were associated with discontinuation of the drug and were independent of renal status. Flu-like symptoms were seen in three of 17 patients enrolled in the study reported by Alsayed et al, but none were greater than grade 2. Additionally, no cardiotoxicity, in particular QTc interval prolongation, has been noted with carfilzomib.

Hematologic adverse events were also considered to be relatively minor. Most are grade 2 or less, and include anemia (29\%-65\%), thrombocytopenia $(25 \%-46 \%)$, and febrile neutropenia $(20 \%-42 \%)$. The overwhelming majority of these events were reversible during periods off the drug. Grade 3 or 4 hematologic toxicity was seen in approximately $9 \%-10 \%$ of the patients. Overall, the spectrum of hematologic toxicities is not unexpected, and well within the realm of what has been previously reported with other proteasome inhibitors.

Some acute noncumulative elevations in creatinine have been seen with the first dose of carfilzomib, but none were irreversible or severe enough to cause toxicity or discontinuation of therapy. Two cases of what appeared to be tumor lysis syndrome were observed early in the PX-171-003-A0 trial, although no additional cases have been seen since routine prophylaxis for tumor lysis was incorporated into subsequent trials.

Interestingly, there have been no reported cases of zoster reactivation with carfilzomib, which has been reported with bortezomib. It is unclear at this time whether this is due to the fact that more patients were initiated on prophylactic antiviral agents pre-emptively, or whether there is a biological basis for the observation. Nonetheless, the absence of zoster reactivation with carfilzomib seen thus far is considered a favorable distinguishing feature of this drug over bortezomib.

\section{Unanswered questions}

While there are clear differences in the biologic effects of carfilzomib compared with the other proteasome inhibitors, such as irreversible proteasome inhibition, greater selective action against the chymotrypsin-like subunit of the proteasome, and more activity against the immunoproteasome, the clinical implications of these differences is only now beginning to be established. Extensive literature has been published documenting the many pleiotropic effects of bortezomib on the bone marrow microenvironment and on the biology of the myeloma cells directly. There is less information regarding the ability of carfilzomib to induce similar effects in these model systems of myeloma.

As already mentioned, it is likely that nonselective proteasome inhibition by bortezomib may contribute to the induction of treatment-emergent neuropathy, which can be dose-limiting. ${ }^{55}$ While both bortezomib and carfilzomib appear to target the $20 \mathrm{Si}$, along with the constitutively active proteasome, it remains to be determined whether carfilzomib 
causes greater selective inhibition of the 20Si subunits, leading to decreased peripheral neuropathy, along with its ability to overcome bortezomib resistance. Fluorogenic enzyme assays to measure quantitative inhibition of the $20 \mathrm{Si}$ subunits are now available, and their incorporation in clinical trials might answer this question.

Differences in the pharmacology, potency, and efficacy across the proteasome inhibitors are incompletely understood. Bortezomib is a small boronic acid dipeptide molecule that mainly inhibits the chymotrypsin-like activity of the $20 \mathrm{~S}$ proteasome. Relative to bortezomib, carfilzomib inhibits the chymotrypsin-like activity of the proteasome more selectively, with less cross-reactivity at the caspase-like and trypsin-like sites. Whether such selective proteasomal inhibition in the context of multiple myeloma makes it more potent and less toxic than NPI-0052, which appears to target all three catalytic sites, remains unknown. The comparative $\mathrm{IC}_{50}$ values of the above three agents for the different catalytic sites of the proteasome are listed in Table 1, and highlight the preferential activity of carfilzomib for the chymotrypsin subunit of the proteasome compared with bortezomib and NPI-0052. It is also possible that the spectrum of proteasome inhibition might dictate the nature of the toxicity profile in patients treated with these compounds. It would be interesting to see if this observation could be exploited to tailor individual therapy in patients with multiple myeloma and other hematologic malignancies without compromising efficacy. Additionally, the timing and method of carfilzomib incorporation in the treatment of patients with multiple myeloma will need to be addressed.

There have been several prospective Phase II trials of bortezomib in patients with relapsed or refractory mantle cell lymphoma, demonstrating overall response rates of $29 \%-50 \%$ and complete response rates of $4 \%-8 \% .^{56-61}$ The median duration of response is approximately 10 months. Adverse events are similar to those previously reported for bortezomib in patients with multiple myeloma. This led to the approval of bortezomib for the treatment of patients with mantle cell lymphoma who have received at least one prior therapy. Obviously, one of the key questions that will need to be answered is whether similar response rates can be achieved with carfilzomib in non-Hodgkin's lymphomas, such as mantle cell lymphoma. Of the five patients with mantle cell lymphoma treated with carfilzomib in the PX-171-001 trial, one patient achieved an unconfirmed overall response. Only one patient with Waldenstrom's macroglobulinemia was included, who showed a $25 \%$ reduction in serum $M$ protein after two cycles of carfilzomib at a dose of $15 \mathrm{mg} / \mathrm{m}^{2}$. In the
PX-171-002 trial, of three patients with mantle cell lymphoma, two achieved stable disease with carfilzomib. While there definitely seems to be evidence of activity of carfilzomib in mantle cell lymphoma, the numbers of patients with this disease entity included in the trials of carfilzomib so far are too small to draw major conclusions at this juncture and need further investigation.

Combinations of bortezomib with other proven agents in relapsed and refractory multiple myeloma have shown promising results. Clinical trials of bortezomib in doublet drug combination regimens (bortezomib + melphalan or bortezomib + dexamethasone) have exhibited overall response rates of $63 \%-70 \%$ with complete response rates of $15 \%-23 \%{ }^{62-64}$ Trials with bortezomib in triplet drug combinations (bortezomib + cyclophosphamide + steroids, bortezomib + adriamycin + steroids, or bortezomib + lenalidomide + steroids) have induced overall response rates of $51 \%-82 \%$, and overall response rates of $15 \%-27 \% .{ }^{65-71}$ Finally, combinations of bortezomib with three other agents active in multiple myeloma have demonstrated overall response rates of $67 \%-92 \%$ and complete response rates of $44 \%-53 \%{ }^{72-75}$ A Phase I trial by Niesvizky et al showed that carfilzomib can be safely combined with lenalidomide and dexamethasone, and induces a high overall response rate $(59 \%-72 \%)$ and complete response rate $(21 \%)$ without increased toxicity. ${ }^{48,49}$ Whether such synergy can be obtained with other chemotherapeutic agents and would be as effective, if not less, compared with bortezomib-based regimens, remains to be determined.

Given the plethora of proteasome inhibitors now being studied in the clinic, clearly it would be of value to be able to define those clinical scenarios where a particular proteasome inhibitor will work. It remains unclear if the activity of these compounds will be limited to myeloma and selected forms of lymphoma, or whether differences in the underlying molecular pharmacology will open new opportunities in other conditions.

Recently, Zangari et al reported that alkaline phosphatase variation during carfilzomib treatment is associated with the best response in multiple myeloma. ${ }^{76}$ They performed a retrospective analysis of serum alkaline phosphatase on 77 patients enrolled in the PX-171-003 and PX-171-004 trials. Increase in alkaline phosphatase from baseline, which was most evident during the second cycle of treatment, was significantly different in patients who achieved equal or greater than a very good partial response compared with all others on days 1 and 8 of cycle 2 . In all patients achieving a very good partial response or better, alkaline 
phosphatase increased by more than $15 \mathrm{U} / \mathrm{L}$ at cycle 2 on day 1 over the baseline. None of the patients with progressive disease exhibited a similar increase, suggesting that this common blood test could be used as a surrogate to predict response.

\section{Conclusion}

It has been approximately 15 years since Julian Adams and his colleagues reported on the original synthesis and activity of bortezomib in in vitro and in vivo models of human cancer. In the past 15 years, our understanding of the ubiquitin-proteasome pathway has become increasingly more sophisticated, now leading to the development of highly targeted drugs capable of targeting discrete ubiquitin E3 ligases. ${ }^{77}$ While the pace of our knowledge in this field continues to grow, there remains many important questions regarding the optimal strategies for use of these agents in the treatment of cancer. For example, is it better to develop more highly selective proteasome inhibitors for one protease over another, or is it better to inhibit some combination of proteases simultaneously? From a pharmacodynamic perspective, is peak concentration or area under the curve of exposure the important determinant in exploiting the clinical activity of these compounds in the clinical setting? Nevertheless, despite multiple unresolved questions with respect to the role of carfilzomib in multiple myeloma and the fact that this is a targeted therapy with a pleiotropic effect, the data to date support the contention that carfilzomib has demonstrated significant clinical activity in patients with relapsed and refractory multiple myeloma, with good durability and tolerable side effects across all stages, irrespective of cytogenetic abnormalities and various degrees of renal impairment. Its marked safety and comparable efficacy in multiple myeloma patients with pre-existing peripheral neuropathy makes it a drug that will integrate well with other active agents. Its efficacy in patients who have become refractory to other proteasome inhibitors, such as bortezomib, gives it a potentially significant role in clinical practice. It is clear that combination drug regimens in multiple myeloma are associated with higher overall and complete response rates, and are capable of inducing rapid response when required. It is safe to assume that future trials of carfilzomib evaluating its role either as a single agent or in combination with other agents, such as immunomodulatory agents and cytotoxic chemotherapy in both frontline and relapsed settings, will emerge in the years to come.

\section{Disclosure}

The authors report no conflicts of interest in this work.

\section{References}

1. Kumar SK, Rajkumar SV, Dispenzieri A, et al. Improved survival in multiple myeloma and the impact of novel therapies. Blood. 2008;111:2516-2520.

2. Kastritis E, Zervas K, Symeonidis A, et al. Improved survival of patients with multiple myeloma after the introduction of novel agents and the applicability of the International Staging System (ISS): An analysis of the Greek Myeloma Study Group (GMSG). Leukemia. 2009;23:1152-1157.

3. Singhal S, Mehta J, Desikan R, et al. Antitumor activity of thalidomide in refractory multiple myeloma. N Engl J Med. 1999;341:1565-1571.

4. Richardson PG, Barlogie B, Berenson J, et al. A Phase 2 study of bortezomib in relapsed, refractory myeloma. N Engl J Med. 2003;348: 2609-2617.

5. Richardson PG, Sonneveld P, Schuster MW, et al. Bortezomib or highdose dexamethasone for relapsed multiple myeloma. $N$ Engl J Med. 2005;352:2487-2498.

6. Rajkumar SV, Hayman SR, Lacy MQ, et al. Combination therapy with lenalidomide plus dexamethasone (Rev/Dex) for newly diagnosed myeloma. Blood. 2005;106:4050-4053.

7. Richardson PG, Blood E, Mitsiades CS, et al. A randomized Phase 2 study of lenalidomide therapy for patients with relapsed or relapsed and refractory multiple myeloma. Blood. 2006;108:3458-3464.

8. Fuchs D, Berges C, Opelz G, et al. Increased expression and altered subunit composition of proteasomes induced by continuous proteasome inhibition establish apoptosis resistance and hyperproliferation of Burkitt lymphoma cells. J Cell Biochem. 2008;103:270-283.

9. Ruckrich T, Kraus M, Gogel J, et al. Characterization of the ubiquitin-proteasome system in bortezomib-adapted cells. Leukemia. 2009;23:1098-1105.

10. Lu S, Chen Z, Yang J, et al. Overexpression of the PSMB5 gene contributes to bortezomib resistance in T-lymphoblastic lymphoma/leukemia cells derived from Jurkat line. Exp Hematol. 2008;36:1278-1284.

11. Oerlemans R, Franke NE, Assaraf YG, et al. Molecular basis of bortezomib resistance: Proteasome subunit beta5 (PSMB5) gene mutation and overexpression of PSMB5 protein. Blood. 2008;112:2489-2499.

12. Lu S, Yang J, Song X, et al. Point mutation of the proteasome beta5 subunit gene is an important mechanism of bortezomib resistance in bortezomib-selected variants of Jurkat T cell lymphoblastic lymphoma/ leukemia line. J Pharmacol Exp Ther. 2008;326:423-431.

13. Moreau P, Pylpenko H, Grosicki S, et al. A Phase 3 prospective randomized international study (MMY-3021) comparing subcutaneous and intravenous administration of bortezomib in patients with relapsed multiple myeloma. Blood. 2010;116:141.

14. Demo SD, Kirk CJ, Aujay MA, et al. Anti-tumor activity of PR-171, a novel irreversible inhibitor of the proteosome. Cancer Res. 2007;67: 6383-6391.

15. Adams J. The proteasome: A suitable antineoplastic target. Nat Rev Cancer. 2004;4:349-360.

16. Orlowski RZ, Kuhn DJ. Proteasome inhibitors in cancer therapy: Lessons from the first decade. Clin Cancer Res. 2008;14:1649-1657.

17. McConkey DJ, Zhu K. Mechanisms of proteasome inhibitor action and resistance in cancer. Drug Resist Updat. 2008;11:164-179.

18. SterzJ, Hahne JC, Lamottke B, et al. The potential of proteasome inhibitors in cancer therapy. Expert Opin Investig Drugs. 2008;17: 879-895.

19. Adams J. The proteasome: Structure, function, and role in the cell. Cancer Treat Rev. 2003;29 Suppl:3-9.

20. Kisselev AF, Callard A, Goldberg AL. Importance of the different proteolytic sites of the proteasome and the efficacy of inhibitors varies with the protein substrate. J Biol Chem. 2006;281:8582-8590.

21. Bennett MK, Kirk CJ. Development of proteasome inhibitors in oncology and autoimmune diseases. Current Opin Drug Discov Devel. 2008; 11:616-625.

22. Altun M, Galardy PJ, Shringarpure R, et al. Effects of PS-341 on the activity and composition of proteasomes in multiple myeloma cells. Cancer Res. 2005;65:7896-7901.

23. Groll M, Kim KB, Kairies N, et al. Crystal structure of epoxomicin: 20S proteasome reveals a molecular basis of $\alpha_{1}, \beta_{1}$-epoxyketone proteasome inhibitors. J Am Chem Soc. 2000;122:1237-1238. 
24. Groll M, Huber R, Potts BC. Crystal structures of salinosporamide A (NPI-0052) and B (NPI-0047) in complex with the 20S proteasome reveal important consequences of $\beta$-lactone ring opening and a mechanism for irreversible binding. J Am Chem Soc. 2006;128:5136-5141.

25. Demo SD, Buchholz TJ, Laidig GJ, et al. Biochemical and cellular characterization of the novel proteasome inhibitor PR-171. Blood. 2005; 106:Abstr 1588

26. Adams J, Behnke M, Chen S, et al. Potent and selective inhibitors of the proteasome: Dipeptidyl boronic acids. Bioorg Med Chem Lett. 1998;8: 333-338.

27. Dorsey BD, Iqbal M, Chatterjee S, et al. Discovery of a potent, selective, and orally active proteasome inhibitor for the treatment of cancer. $J$ Med Chem. 2008;51:1068-1072.

28. Parlati F, Lee S, Aujay, et al. Carfilzomib can induce tumor cell death through selective inhibition of the chymotrypsin-like activity of the proteasome. Blood. 2009;114:3439-3447.

29. Kuhn DJ, Chen Q, Voorhees, et al. Potent activity of carfilzomib, a novel irreversible inhibitor of the ubiquitin-proteasome pathway, against preclinical models of multiple myeloma. Blood. 2007;110:3281-3290.

30. Suzuki E, Demo S, Arastu-Kapur S, et al. Bortezomib resistant cell lines have increased proteasome levels but remain sensitive to carfilzomib. Blood. 2009;114:Abstr 2852.

31. Wang L, Kumar S, Fridley BL, et al. Proteasome $\beta$ subunit pharmacogenomics: Gene resequencing and functional genomics. Clin Cancer Res. 2008; 14:3503.

32. Trudel S, Lee S, Kirk CJ, et al. Inhibition of the proteasome in bone marrow derived $\mathrm{CD} 138^{+}$tumor cells following carfilzomib administration in relapsed and refractory myeloma patients. Blood. 2009;114:Abstr 1845.

33. Stapnes C, Doskeland A, Hatfield K, et al. The proteasome inhibitors bortezomib and PR-171 have antiproliferative and proapoptotic effects on primary human acute myeloid leukemia cells. $\mathrm{Br} J$ Haematol. 2007;136:814-828.

34. Dasmahapatra G, Lembersky D, Kramer L, et al. The pan-HDAC inhibitor vorinostat potentiates the activity of the proteasome inhibitor carfilzomib in human DLBCL cells in vitro and in vivo. Blood. 2003; $115: 4478-4487$.

35. Rao R, Fiskus W, Balusu R, et al. Treatment with histone deacetylase-6 inhibitor WT-161 disrupts hsp-90 function, abrogates aggresome formation and sensitizes human mantle cell lymphoma cells to lethal ER stress induced by proteasome inhibitor carfilzomib. Blood. 2010;116:Abstr 2856.

36. Kirk CJ, Benett MK, Buchholz TJ, et al. Pharmacokinetics, pharmacodynamics and anti-tumor efficacy of PR-171, a novel inhibitor of the 20S proteasome. Blood. 2005;106:Abstr 609.

37. O'Connor OA, Stewart AK, Vallone M, et al. A Phase I dose escalation study of the safety and pharmacokinetics of the novel proteasome inhibitor carfilzomib (PR-171) in patients with hematologic malignancies. Clin Cancer Res. 2009;15:7085-7091.

38. Rosen PJ, Gordon M, Lee PN, et al. Phase II results of Study PX-171007: A Phase Ib/II study of carfilzomib, a selective proteasome inhibitor, in patients with selected advanced metastatic solid tumors. J Clin Oncol. 2009;27 Suppl:3515.

39. Lee P, Wong AF, Burris HA, et al. Updated results of a Phase Ib/II study of carfilzomib in patients with relapsed malignancies. $J$ Clin Oncol. 2010;28(15Suppl):8147.

40. Alsina M, Trudel S, Vallone M, et al. Phase I single agent antitumor activity of twice weekly consecutive dosing of the proteasome inhibitor carfilzomib (PR-171) in hematologic malignancies. Blood. 2007; 110:Abstr 411.

41. Jagannath S, Vij R, Stewart K, et al. Final results of PX-171-003-A0, part 1 of an open label, single arm, Phase II study of carfilzomib (CFZ) in patients with relapsed and refractory multiple myeloma. J Clin Oncol. 2009;27(15 Suppl):8504.

42. Siegel DS, Martin T, Wang M, et al. Results of PX-171-003-A1, an open label, single arm, Phase 2 study of carfilzomib in patients with relapsed and refractory multiple myeloma. Blood. 2010;116: Abstr 985.
43. Vij R, Wang M, Orlowski R, et al. PX-171-004, a multicenter Phase II study of carfilzomib in patients with relapsed myeloma: An efficacy update. J Clin Oncol. 2009;27(15 Suppl):8537.

44. Wang L, Siegel D, Kaufman JL, et al. Updated results of bortezomibnaive patients in PX-171-004, an ongoing open label, Phase II study of single agent carfilzomib in patients with relapsed or refractory MM. Blood. 2009;114:Abstr 302.

45. Siegel D, Wang L, Orlowski RZ, et al. PX-171-004, an ongoing open label Phase II study of single agent carfilzomib in patients with relapsed or refractory MM; updated results from the bortezomib treated cohort. Blood. 2009; 114:Abstr 303.

46. Vij R, Siegel DS, Kaufman JL, et al. Results of an ongoing open label, Phase II study of carfilzomib in patients with relapsed and/or refractory MM. J Clin Oncol. 2010;28(15 Suppl):8000.

47. Jakubowiak A, Wang L, Orloski R, et al. Influence of cytogenetics in patients with relapsed and refractory multiple myeloma treated with carfilzomib. Blood. 2009;114:Abstr 1827.

48. Niesvizky R, Bensinger W, Vallone M, et al. PX-171-006: Phase Ib multicenter dose escalation study of carfilzomib plus lenalidomide and low-dose dexamethasone in relapsed and refractory MM: Preliminary results. J Clin Oncol. 2009;27(15 Suppl):8514.

49. Bensinger W, Wang M, Orlowski RZ, et al. Dose escalation study of carfilzomib plus lenalidomide plus low-dose dexamethasone in relapsed and refractory MM. J Clin Oncol. 2010;28(15 Suppl):8029.

50. Alsayed Y, Nair BP, Kauffman M, et al. Carfilzomib in combination with Dex and other agents (Dox, thal, DDP, vorinostat ) for far advanced and refractory multiple myeloma (FARMM). J Clin Oncol. 2010;28(15 Suppl):e18504

51. Badros A, Vij R, Martin T, et al. Phase I study of carfilzomib in patients with relapsed and refractory multiple myeloma and varying degrees of renal insufficiency. Blood. 2009;114:Abstr 3877.

52. Badros A, Vij R, Martin T, et al. Phase II study of carfilzomib in patients with relapsed/refractory multiple myeloma and renal insufficiency. J Clin Oncol. 2010;28(15 Suppl):8128.

53. Vij R, Wang L, Orlowski, et al. Carfilzomib, a novel proteasome inhibitor for relapsed or refractory multiple myeloma, is associated with minimal peripheral neuropathic effects. Blood. 2009;114:Abstr 430.

54. Wolf JL, Vij R, Lonial S, et al. Neurotoxic and peripheral neuropathic beffects in preclinical and clinical studies of carfilzomib, a novel proteasome inhibitor. J Clin Oncol. 2010;28(15 Suppl):8135.

55. Kuhn DJ, Hunsucker SA, Voorhees PM, et al. Targeted inhibition of the proteasome is a potent strategy against models of myeloma that overcome resistance to conventional drugs and nonspecific proteasome inhibitors. Blood. 2009;113:4667-4676.

56. Goy A, Younes A, McLaughlin P, et al. Phase II study of proteasome inhibitor bortezomib in relapsed or refractory B-cell non-Hodgkin's lymphoma. J Clin Oncol. 2005;23:667-675.

57. O'Connor OA, Wright J, Moskowitz C, et al. Phase II clinical experience with the novel proteasome inhibitor bortezomib in patients with indolent non-Hodgkin's lymphoma and mantle cell lymphoma. $J$ Clin Oncol. 2005;23:676-684.

58. Strauss SJ, Maharaj L, Hoare S, et al. Bortezomib therapy in patients with relapsed or refractory lymphoma: Potential correlation of in vitro sensitivity and tumor necrosis factor alpha response with clinical activity. J Clin Oncol. 2006;24:2105-2112.

59. Fisher RI, Bernstein SH, Kahl BS, et al. Multicenter Phase II study of bortezomib in patients with relapsed or refractory mantle cell lymphoma. J Clin Oncol. 2006;24:4867-4874.

60. Belch A, Kouroukis CT, Crump M, et al. A Phase II study of bortezomib in mantle cell lymphoma: The National Cancer Institute of Canada Clinical Trials Group trial IND.150. Ann Oncol. 2007;18:116-121.

61. Gerecitano J, Portlock C, Moskowitz C, et al. Phase 2 study of weekly bortezomib in mantle cell and follicular lymphoma. $\mathrm{Br} J$ Haematol. 2009; 146:652-655.

62. Berenson JR, Yang HH, Vescio RA, et al. Safety and efficacy of bortezomib and melphalan combination in patients with relapsed or refractory multiple myeloma: Updated results of a Phase 1/2 study after longer follow-up. Ann Hematol. 2008;87:623-631. 
63. Popat R, Oakervee H, Williams C, et al. Bortezomib, low-dose intravenous melphalan, and dexamethasone for patients with relapsed multiple myeloma. Br J Haematol. 2009;144:887-894.

64. Pineda-Roman M, Zangari M, van Rhee F, et al. VTD combination therapy with bortezomib-thalidomide-dexamethasone is highly effective in advanced and refractory multiple myeloma. Leukemia. 2008;22:1419-1427.

65. Reece DE, Piza G, Trudel S, et al. A Phase I-II trial of bortezomib plus oral cyclophosphamide and prednisone for relapsed/refractory multiple myeloma. Blood. 2006;108:Abstr 3536.

66. Kropff M, Bisping G, Schuck E, et al. Bortezomib in combination with intermediate-dose dexamethasone and continuous low-dose oral cyclophosphamide for relapsed multiple myeloma. $\mathrm{Br} J$ Haematol. 2007; 138:330-337.

67. Hajek R, Zahradova L, Gregora E, et al. The reduced intensity CVD regimen: A good option with well-balanced efficacy/toxicity ratio for elderly patients with poor status performance. Blood. 2008;112:Abstr 3699.

68. Lee SS, Suh C, Kim BS, et al. Bortezomib, doxorubicin and dexamethasone (PAD) combination therapy followed by thalidomide and dexamethasone (TD) as a salvage treatment for relapsed multiple myeloma (MM): Preliminary analysis of efficacy and safety. Blood. 2007;110:Abstr 2731.

69. Palumbo A, Gay F, Bringhen S, et al. Bortezomib, doxorubicin and dexamethasone in advanced multiple myeloma. Ann Oncol. 2008;19: $1160-1165$.

70. Richardson P, Jagannath S, Jakubowiak A, et al. Lenalidomide, bortezomib, and dexamethasone in patients with relapsed or relapsed/ refractory multiple myeloma (MM): Encouraging response rates and tolerability with correlation of outcome and adverse cytogenetics in a Phase II study. Blood. 2008;112:Abstr 1742.
71. Poenisch W, Bourgeois M, Wang SY, et al. Bortezomib in combination with bendamustine and prednisone in the treatment of patients with refractory/relapsed multiple myeloma. Blood. 2007;110: Abstr 2723.

72. Palumbo A, Ambrosini MT, Benevolo G, et al. Bortezomib, melphalan, prednisone, and thalidomide for relapsed multiple myeloma. Blood. 2007;109:2767-2772.

73. Terpos E, Heath DJ, Zervas K, Dimopoulos MA. The effect of bortezomib monotherapy and bortezomib-based regimens on bone metabolism in patients with relapsed/refractory multiple myeloma. Paper presented at: Haematologica. 2007;92:133 (PO320). [4th International Workshop on Waldenstrom's macroglobulinaemia. Kos, Greece, 25-30/6/2007].

74. Ciolli S, Leoni F, Casini C, Breschi C, Santini V, Bosi A. The addition of liposomal doxorubicin to bortezomib, thalidomide and dexamethasone significantly improves clinical outcome of advanced multiple myeloma. Br J Haematol. 2008;141:814-819.

75. Kim YK, Lee JJ, Sohn SK, et al. Clinical efficacy of VEL-CTD (bortezomib, cyclophosphamide, thalidomide, and dexamethasone) regimen in patients with relapsed or refractory multiple myeloma: A Phase II study. Blood. 2008;112:Abstr 3693.

76. Zangari M, Polavaram L, Zhan F, et al. Alkaline phosphatase variation during carfilzomib treatment is associated to best response in multiple myeloma. Blood. 2009; 114:Abstr 2895.

77. Chen Q, Xie W, Kuhn DJ, et al. Targeting the p27 E3 ligase SCF ${ }^{\text {Skp2 }}$ results in p27- and Skp2-mediated cell-cycle arrest and activation of autophagy. Blood. 2008;111:4690-4699.

78. Richardson PG, Hofmeister C, Jakubowiak A, et al. Phase 1 clinical trial of the novel structure proteasome inhibitor NPI-0052 in patients with relapsed and relapsed/refractory multiple myeloma (MM). Blood. 2009; 114:Abstr 431.

\section{Core Evidence}

\section{Publish your work in this journal}

Core Evidence is an international, peer-reviewed open-access journal evaluating the evidence underlying the potential place in therapy of drugs throughout their development lifecycle from preclinical to postlaunch. The focus of each review is to evaluate the case for a new drug or class in outcome terms in specific indications and patient groups

\section{Dovepress}

The manuscript management system is completely online and includes a very quick and fair peer-review system, which is all easy to use. Visit http://www.dovepress.com/testimonials.php to read real quotes from published authors. 